\section{COPD: Welche Antihypertensiva sind vorteilhaft, welche eher nicht?}

Die COPD ist eine der häufigsten Begleiterkrankungen bei Hypertonikern. Und diese Komorbidität hat Auswirkungen auf die medikamentöse antihypertensive Therapie; denn einige Antihypertensiva sind ungünstig für die Lunge, andererseits kann auch die COPD-Therapie den Blutdruck, genauer gesagt die Wirkung der Antihypertensiva ungünstig beeinträchtigen.

$\mathrm{D}$ ie häufigste Begleiterkrankung bei Patienten mit einer chronisch obstruktiven Lungenerkrankung (COPD) ist mit etwa $28 \%$ die arterielle Hypertonie gefolgt vom Diabetes mellitus und der KHK. Dabei stellt die COPD einen eigenständigen Risikofaktor für die KHK dar. „Bei dieser Komorbidität besteht ein besonders hohes Risiko für ein kardiovaskuläres Ereignis, genauer gesagt ist es um das zwei- bis dreifache erhöht", erläuterte Prof. Csaba Farsang aus Budapest. Für jede dieser beiden Erkrankungen gebe es eine eigene Leitlinie, aber nicht für die Komorbidität. Doch bei der medikamentösen Therapie müsse bei solchen Patienten einiges bedacht werden, da Antihypertensiva die Lungenfunktion zusätzlich verschlechtern und umgekehrt COPD-Medikamente die Wirkung der Antihypertensiva abschwächen können.

\section{Diuretika sind oft ungünstig}

Diuretika gelten als idealer Kombinationspartner bei Gabe eines RAAS-Hemmers. Dies gilt aber nicht für COPD-Pa- tienten. Doch bei einer Herzinsuffizienz mit Stauung sind sie unverzichtbar. Allerdings senken sie den Kaliumspiegel und können die durch Beta-2-Agonisten und Steroide induzierte Hypokaliämie noch verstärken, mit den sich daraus ergebenden arrhythmogenen Risiken.

Thiazide entfalten darüber hinaus weitere ungünstige Effekte. Sie können die $\mathrm{CO}_{2}$-Retention und die metabolische Alkalose und somit die Hypoxie bei Hypoventilation verstärken, sie erhöhen den Hämatokrit und sie verändern die Schleimbildung, sodass ein Abhusten erschwert wird. „Deshalb wird die Gabe eines Diuretikums ausschließlich zur Blutdrucksenkung bei COPD-Patienten nicht unbedingt empfohlen", so Farsang.

Für Indapamid konnte allerdings bei hypertensiven COPD-Patienten neben der Blutdrucksenkung auch eine Verbesserung der Lungenfunktion dokumentiert werden. Bei herzinsuffizienten $\mathrm{Pa}$ tienten können Diuretika, auch wenn gleichzeitig eine COPD vorliegt, das Risiko für eine erneute stationäre Behand-

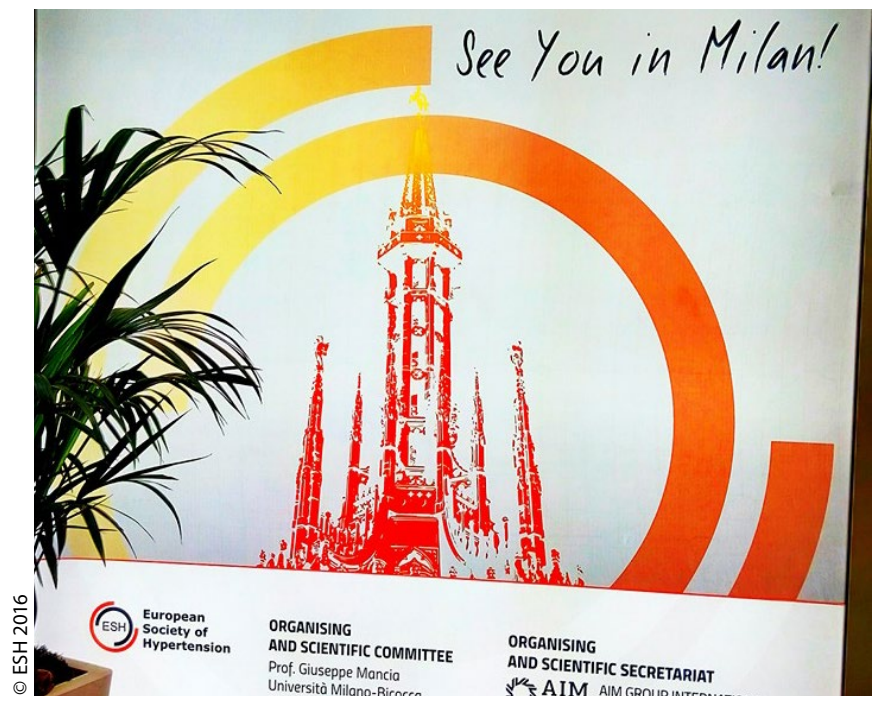

Zum nächsten Kongress begrüßt Sie die European Hypertension Society (ESH) 2017 in Mailand. lung verringern. Auch dürften, was den Kaliumspiegel betrifft, kaliumsparende Diuretika wie Amilorid bzw. Aldosteron-Antagonisten günstiger sein.

\section{Keine Kontraindikation für Betablocker}

Bei Patienten mit einer COPD können bzw. sollten sogar Betablocker eingesetzt werden, aber nur kardioselektive oder solche, die auch eine leichte Beta-2-agonistische Wirkkomponente aufweisen oder die NO-Produktion steigern wie Nebivolol. Solche Substanzen können sogar das Exazerbationsrisiko verringern. Sie verstärken die bronchodilatierende Wirkung der Beta-2-Agonisten, da sie zu einer Aufregulation der Beta-2-Rezeptoren führen. Und bei kardial dekompensierten Patienten wird durch den Betablocker sogar die Prognose verbessert.

Kurzum die Überlebenschance korrelierte in den entsprechenden Studien mit der Einnahme eines Betablockers. Auch für Carvedilol, welches einen nicht-selektiven Betablocker darstellt, aber auch eine Adrenorezeptoren-blockierende Wirkung entfaltet, konnte bei COPD-Patienten keine Verschlechterung der Lungenfunktion nachgewiesen werden.

Günstig für COPD-Patienten sind Kalziumantagonisten. Sie reduzieren den Tonus der glatten Muskulatur, wodurch die medikamentöse Bronchodilatation verstärkt wird. Doch selten verschlechtert sich darunter der PerfusionVentilations-Quotient, sodass Atemnot und Hypoxie zunehmen können.

Auch ACE-Hemmer sind günstig. Allerdings können sie einen quälenden Hustenreiz und sogar einen Asthmaanfall auslösen. Auch wirken sie dem hypokaliämischen Effekt der Beta-2-Agonisten entgegen. Besser sind deshalb AT1-Blocker. Sie induzieren keinen Hustenreiz und das Risiko für ein angioneurotisches Ödem ist äußerst niedrig.

Ohne Probleme können bei COPDPatienten auch Urapidil, Moxonidin und Alphablocker eingesetzt werden. „Auch wenn das pharmakologische Profil bekannt ist, lässt sich im Einzelfall bei Gabe einer bestimmten Substanz die Wirkung auf die Lunge bei COPD-Patienten nicht immer mit letzter Sicherheit voraussagen", so Farsang.

Dr. med. Peter Stiefelhagen 